\title{
Real-time resource efficiency Indicators
}

Material and energy efficient plant operation

\begin{abstract}
Operational decisions in the day-to-day business of chemical production processes can have a significant impact on the energy and material efficiency. We propose to use real-time resource efficiency indicators (REI) to accurately monitor the energy and material efficiency in real-time and subsequently to use these in decision support for the operating staff. To guide industrial users during the development of REIs, a Namur ad-hoc working group (AK) Resource Efficiency Indicators for the operational use was set up to prepare a Namur Recommendation.
\end{abstract}

\section{KEYWORDS resource efficiency / energy efficiency / performance monitoring / decision support / performance indicators / KPI}

\section{Entwicklung von Echtzeit-Ressourceneffizienz-Indikatoren - Nachhaltige Anlagenfahrweise und Standardisierung}

Operative Entscheidungen können im Tagesgeschäft chemischer Produktionsprozesse einen signifikanten Einfluss auf die Energie- und Materialeffizienz der Anlage haben. Dieser Beitrag beschreibt wie die Energie- und Materialeffizienz auf Basis von Echtzeit-Ressourceneffizienz-Indikatoren (REI) online überwacht und diese Kennzahlen genutzt werden können, um eine Entscheidungsunterstützung für das Betriebspersonal bereitzustellen. Um industrielle Anwender bei der Erarbeitung von REI für ihre Prozesse zu unterstützen, erarbeitet der Namur-Ad-Hoc-AK RessourceneffizienzIndikatoren für den operativen Einsatz eine entsprechende Namur-Empfehlung.

\section{SCHLAGWÖRTER Ressourceneffizienz / Energieeffizienz / Performance-überwachung / Entscheidungsunterstützung / Performance-Indikatoren / KPI}


MARC KALLISKI, Technische Universität Dortmund BENEDIKT BEISSHEIM, IneoS

DANIEL KRAHÉ, Technische Universität Dortmund

UDO ENSTE, LeiKon

STEFAN KRÄMER, Ineos

SEBASTIAN ENGELL, Technische Universität Dortmund

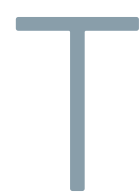

he efficient use of energy in chemical production processes was at the centre of optimisation efforts in recent years and led to efficiency analysis systems for energy utilization, such as the Structese programme [1]. Experts in the field of energy management still see a high potential for additional energy conservation and a need for concise key performance indicators (KPI) to assess the plant efficiency on short timescales and to overcome limitations of current energy performance indicators (EnPIs) [2]. The motivation for the implementation of EnPIs in the German process industry usually is the interest to establish an energy management system that is compliant with ISO 50001:2011 [3] which results in many advantages besides the reduction of the consumption of energy, including exemption from energy audits or possible tax reductions. Usually the EnPIs that are required for certified energy management systems only consider energy imports across the system boundary while neglecting, for example, energy supply through raw materials, as is common in the petrochemical industry and in refineries. Therefore, we propose to use Resource Efficiency Indicators (REIs) that capture energy efficiency and material efficiency, can be used to guide the daily operation of a plant, and reflect the overall efficiency as a multi-dimensional entity [4].

The material and energy efficiency of chemical production processes is strongly influenced by the operational decisions made during daily production. The complexity and the high degree of integration of the various plants and units causes coupling due to stoichiometry, heat integration and recycling streams, resulting in causal relationships and trade-offs beyond the performance of a single unit that are not always obvious to the operators. For instance, a locally optimized unit in plant A can lead to a high energy demand in plant $B$ due to heat integration and can subsequently result in a sub-optimal state of the overall production site (cf. fig. 1).

In the EU project Real-time Monitoring and Optimization of Resource Efficiency in Integrated Processing Plants (MORE), funded under the seventh framework programme of the EU, No. 604068, a structured metho- dology to define real-time REI has been developed. The visualization of the REI enables operators and plant managers to identify situations where energy and/or material are not used efficiently and to perform a rootcause analysis. Moreover, operational policies can be optimized.

\section{REAL-TIME RESOURCE EFFICIENCY INDICATORS}

In a first step, the consumption of material and energy, and the output of products are measured over appropriate intervals of time (minutes to hours) for all relevant subunits. If a unit or plant delivers several products, the material and energy input is distributed over the products based on mass and energy balances. The indicators are specific for a product of a given specification, e.g. purity or colour, as different products may require different inputs of energy and material. The indicators can be normalized to the theoretical optimum or historically best observed values. Indicators on the strategic level are calculated by vertical aggregation to capture the overall resource efficiency, in figure 1 this would mean to find the site REIs for plants $\mathrm{A}$ and $\mathrm{B}$.

\subsection{Types of Key Performance Indicators}

The REIs can be divided into two groups (figure 2 left). The first group is based on a material and energy flow analysis (MFA and EFA). Indicators from this group measure how much energy and raw materials are consumed in the production of one unit of product. The second set of indicators measure the environmental impact of the production process. The distinction between these groups is made because the former reflect the efficiency with which resources (materials and energy) are converted into valuable products while the latter additionally measure the impact of possibly small streams, e.g. due to the toxicity of waste streams or a contamination of the product. 


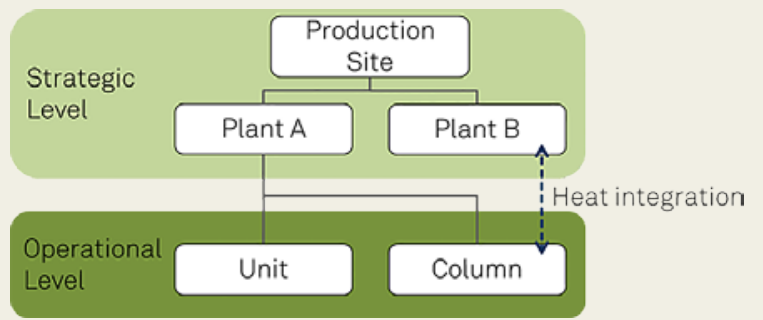

TABLE 1: Aspects to be considered during the definition of REl

FIGURE 1: Coupled production between plants A and B depicted hierarchically.

Overall optimum of Site is desired.

\begin{tabular}{|l|l|l|}
\hline Inputs & Conversions/Mixing/Splitting & Outputs \\
\hline Raw materials & Chemical reactions (mass) & Products \\
Energy by sources & Heat of reaction (energy $\leftrightarrow$ mass) & By-products \\
Other: water, air & $\begin{array}{l}\text { Combustion (energy } \leftrightarrow \text { mass) } \\
\text { Stream splits, mixing }\end{array}$ & $\begin{array}{l}\text { Waste (liquid/solid/gas) } \\
\text { Heat/Energy (losses) }\end{array}$ \\
& (Imperfect) separations & Other emissions \\
\hline
\end{tabular}

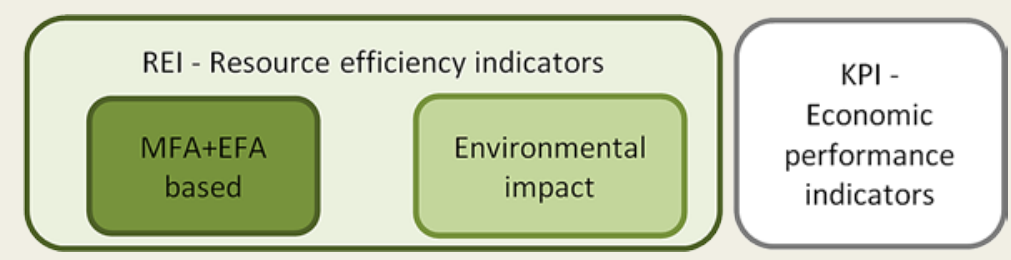

\author{
FIGURE 2: REI considered \\ as physical real-time \\ indicators vs. $\mathrm{KPI}$ as \\ economic key perfor- \\ mance indicators.
}

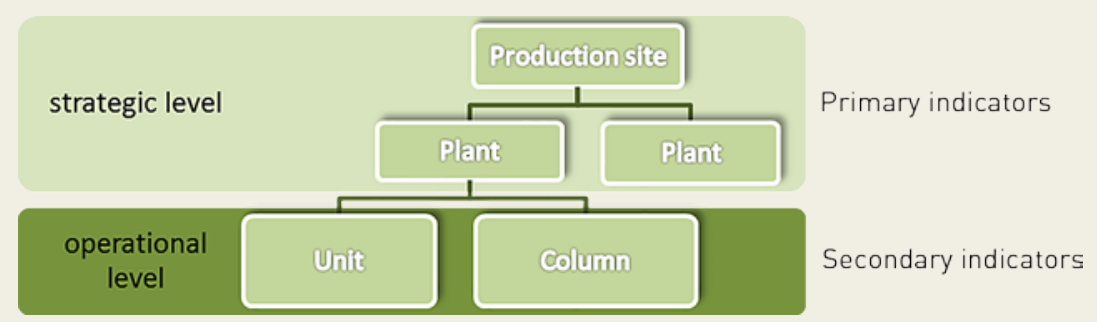

FIGURE 3: Primary indicators have a global meaning with possible strategic importance and it must be possible to aggregate them to a site level. Secondary indicators can be specific to the operation of a unit.

Complementary to the Resource Efficiency Indicators, economic indicators are used in most production facilities to assess the economic performance on various time scales. In most cases, a more resource efficient operation is also economically advantageous, but it is also possible that the two objectives conflict. From the perspective of resource efficiency, for example, the minimization of all waste streams is desirable, but this can entail higher costs, resulting in economically suboptimal production. In such conflicting cases, REI and economic performance indicators should be considered separately, for example in the form of a Pareto curve or Pareto surface that displays those combinations of indicators where one indicator can only be improved at the expense of worsening another. Generally, the trade-off between resource efficiency and economic efficiency must be handled by the company management as a strategic decision, e.g. to produce at a higher cost in order to achieve a smaller $\mathrm{CO}_{2}$-footprint.

\subsection{Principles for Resource Efficiency Indicators}

The methodology that was developed in the project MORE is intended to define REIs that measure the entire resource efficiency for industrial production sites in an objective fashion such that the effect of technical improvements and operational policies on the resource efficiency can be evaluated and actions can be derived to improve the performance of the plant. To achieve this, the indicators must comply with the following eight principles.

\section{Hierarchy of indicators}

If various pieces of equipment of a process are interconnected in a complex fashion as shown in figure 3, an REI for an individual apparatus may be misleading because resource utilization can be shifted to another unit by different local operational policies. Thus, the primary indicators are defined with a global meaning and in a way that they can be aggregated to the highest me- 
aningful level of the hierarchy. In order to improve the overall resource efficiency, which is monitored by the primary REIs, secondary indicators can be introduced on the operational level. Local REIs with a direct meaning and relevance for the operators are useful and important in daily operations. These secondary indicators must be in line with the primary indicators and should not lead to attempts to improve the local performance at the expense of the overall performance. Transparent aggregation and calculation of the contributions of the local units to the global indicators is required to avoid this problem.

\section{Gate-to-gate approach}

The boundary of the analysis is the border of the production site, plant or process unit under consideration, because plant operations can only influence the resource efficiency within these boundaries (gate-to-gate approach). The procurement of more environmentally friendly raw materials or the distribution to more or less sustainably operating customers is a business decision and is not influenced by the decisions of plant operators or plant managers, and hence including these contributions in the spirit of life cycle assessment would add significant influences beyond their control. Nevertheless, aggregation and extension to a full life cycle assessment is possible and intended.

\section{Resource and output specific for meaningful aggregation}

Firstly, the system boundaries for the computation of the primary REIs must be defined. The size of the elements considered should be such that the indicators are unambiguous, i.e. it is clear that improvements of the indicators demonstrate better process performance and are not possibly outweighed by negative effects on subsequent steps or process cycles . Therefore the entities considered should be larger units with input and output streams that lead to a specified product, not individual pieces of equipment. If indicators for subunits are required, they must be comparable and it must be possible to aggregate them to an indicator of the whole unit. Only in this way the influence of the subunit on the total and on a parallel unit can be visualised.

Secondly, all net flows of raw materials, energy, and products that cross the boundaries of the system under consideration have to be determined. Each resource should be measured individually, without aggregation at this stage. In the next step, the specific flows and conversions inside the system limits are analysed in order to link the inputs to the outputs. An exemplary list of streams and conversions that have to be considered is shown in Table 1.

Based on the material and energy flow analysis, process specific REI should be defined with respect to the resources and the products.

$R E I_{R P S}=\frac{\text { Resource Input }}{\text { Product Output }}$
Such a resource and product specific (RPS) REI by itself does not show directly whether the process is operated well, because there is no basis for what is good or bad. Therefore, the REI should be related to a reference case that is obtained from a theoretical analysis (based on mass and energy balances, e.g. stoichiometries) or from the best demonstrated practice (based on historical data). The reference case may and often must vary with external factors such as ambient conditions and plant load in order to yield a trusted indicator that measures the relative performance under given external conditions beyond influence.

$R E I_{\text {norm }}=\frac{R E I_{R P S}}{R_{R P S} \text { reference }}$

\section{Indicators of technical performance}

In the indicators, the flows of material and energy are not to be related to economic indicators such as the sales value of the products or the prices of the resources. As a result, the technical performance is separated from the economic performance. The possibility of a trade-off between resource efficiency and economic performance was discussed above and should be approached, if necessary, by providing the information transparently, e.g. by the computation of Pareto fronts, to allow strategic decisions resolve the conflict.

\section{Based on material and energy flow analysis}

The primary REIs are based on the physical flows and conversion of raw materials and energy to products and outflows to the environment which are objective characteristics of a production process independent of the extrinsic, possibly volatile economic conditions. The important streams for the material and energy flow analysis have to be identified individually for each case. Ideally the material and energy balances are completely closed. If the same input material can be converted into product or converted into by-products or serves as a source of energy, then an integrated energy and material flow analysis must be performed. Different sources of energy can be aggregated using suitable units, e.g. total energy or an energy currency that reflects the efficiency with which, for example, electric power is generated from a primary source of energy.

\section{Considering storage effects}

In the definition of real-time REIs, the choice of the temporal aggregation interval is crucial. On the one hand, the interval should be short so that the indicators can be used to support operational decisions. On the other hand, storage effects should not lead to wrong indications. If material or energy is stored in the unit for a period of time and the amount that is stored varies, then either the indicators have to be computed over periods of time during which the storage effects can be neglected or, preferably, the effect has to be considered in the consumption or production figures. In addition, long-term effects such as catalyst degradation or fouling must either be used in the calculation period or defined as a state for best demonstrated practice in a suitable manner. 


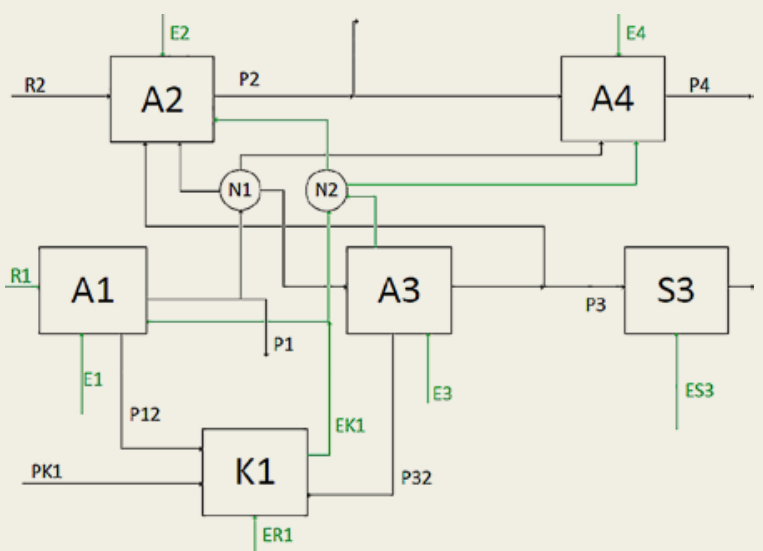

FIGURE 4: Example of a production complex with 5 production units and a power plant

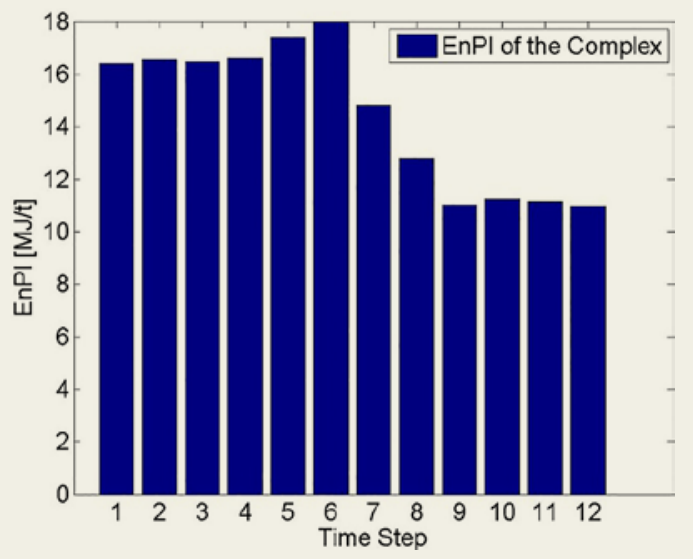

FIGURE 5: Variation of the overall energy efficiency performance of the plant in Figure 4 over time

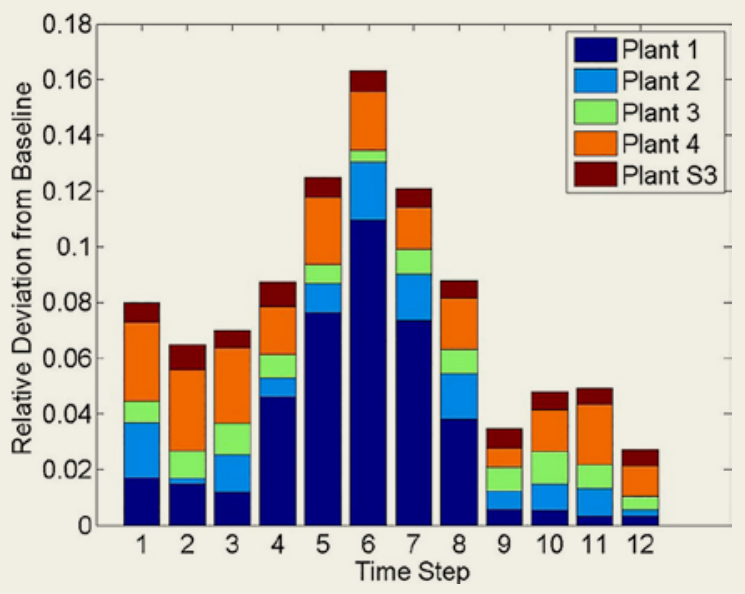

FIGURE 6: Plant-wise relative deviation from the performance baseline over time for the example in Figure 4.

\section{Include environmental impact}

The impact on the environment must be taken into account separately to measure the environmental performance. A full EFA and MFA will include energy losses and pollutant streams. Emission of pollutants to air, water and soil as well as the generation of solid waste per unit of product should be used as separate indicators because their contribution to the energy and mass balances is often very small but nonetheless their impact is significant. It must be considered for each case which categories are needed dependent on the impact and the use of the waste streams. The amount of waste per product manufactured may have to be differentiated according to waste that is incinerated (and recovers or consumes energy), is re-used, e.g. as construction material, or is disposed of. Defining an indicator for the amount of emissions and waste is the basis for evaluation of environmental impacts.

\section{Extensible to life-cycle analysis}

For the operational decisions in a processing plant, upstream or downstream influences may or may not be meaningful. On the one hand, the origin of oils from natural resources may, for example, influence the carbon footprint of the final product, but this can neither be influenced by the technology of the plant nor by its operation, although it could yield a different baseline. The use of more environmentally friendly raw materials may reduce the resource efficiency of the production unit, because of a higher energy input needed in purification steps. On the other hand, the carbon footprint may influence decisions on the use of renewable sources of energy vs. fossil fuels. By initially performing the analysis on a detailed level (i.e. separating the use of electricity and natural gas rather than only the consolidated consumption of energy), it is possible to consider such external effects by relating the streams of mass and energy to environmental indicators in terms of the amount of $\mathrm{CO}_{2}$ produced per unit of the resource. For reporting and assessment purposes, the REIs defined and used in MORE can be extended to a Life Cycle Assessment (e.g. carbon footprint) by weighting the different streams with the upstream impacts.

\section{HIERARCHICAL AGGREGATION SCHEME}

The use of indicators is beneficial on every level of the hierarchy within a company. While indicators for specific plant equipment help the operators to optimize the current operation point, indicators for a plant as a whole are mandatory for reporting and compliance with legal obligations. By computing all mass and energy flows into and out of the balance, reporting with a consistent set of indicators is possible. However, experience shows that large balance domains often suffer from strong fluctuations of the indicators without a clear attribution to root causes. Thus, a generic aggregation approach to compute REIs by a bottom-up me- 

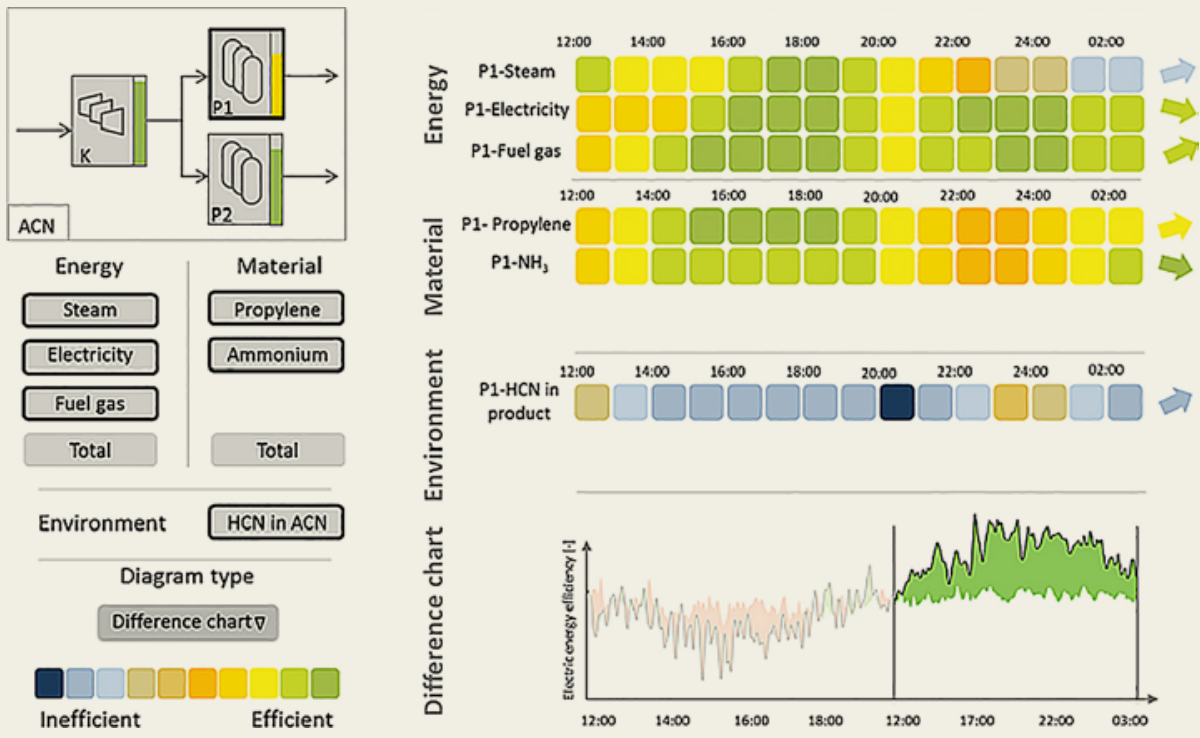

Inefficient

Efficient

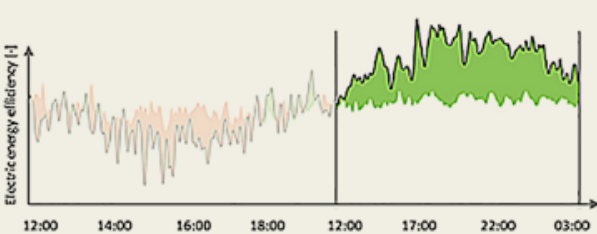

FIGURE 7: Dashboard

for the acrylonitrile

plant operated by

Ineos in Cologne

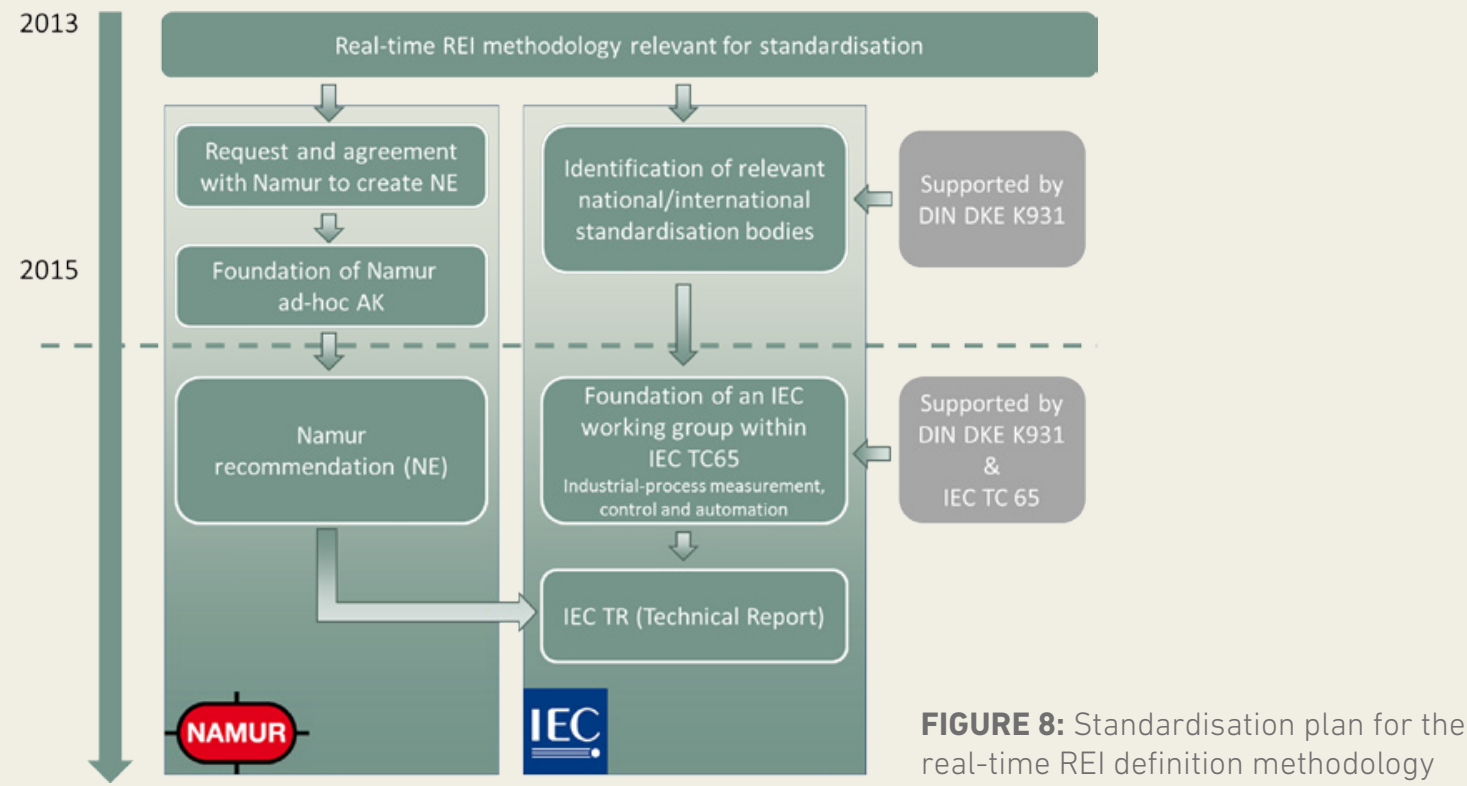

thod was developed within the MORE project. Subunits of a production plant are considered as the lowest level of aggregation. Going down to the equipment level is only useful if all the equipment is well-instrumented, which is not the case in most applications.

Exploiting the information on the structure of the plant, the contribution of each subunit to the aggregated unit becomes apparent. This helps to quickly allocate the source of the deviation from the expected behaviour. In addition to the aggregation of the contribution of each subunit to the overall indicator, the aggregation of the corresponding baselines is also necessary. If the performance of the subunits depends on individual loads and fluctuates frequently, computing the performance of the aggregated unit is not sufficient to evaluate how the unit as a whole is performing. The parallel aggregation of the baselines leads to an objective evaluation of the performance of the overall unit. Computing the contribution of each subunit to the deviation of the performance of the overall unit from the baseline allows operators and ma- 
nagers to quickly identify sources of problems and areas where improvements should be targeted.

Figure 4 shows an example of an integrated chemical park with five production units (A1-A4, S3), a power plant (K1), and two networks (N1, N2). Figure 5 shows a time series of the aggregated energy performance of all production plants on the site in energy per unit mass of product. The indicator increases during the first six time steps, then decreases significantly during the next three time steps before settling at a constant level that is below the initial level.

It seems that the energy performance of the site slightly decreased before increasing significantly. But the reason for this behaviour remains unclear. The evaluation of the contribution of each plant to the deviation from the aggregated baseline reveals the reason for this behaviour (see fig. 6). During time steps 4-8, plant 1 contributed significantly more to the deviation from the baseline, while the contribution of the other plants remained nearly constant. In time steps 9-12, the contribution of plant 1 is comparable to steps 1-3. During steps 4-6, plant 1 consumed more energy than required by the baseline. In steps 7-9, the production rate was increased, which required more energy for three time steps. After this decrease, the performance of the plant returned to its former level while the total production rate of the complex increased. As the production rate is in the denominator of the indicator, an increase of production reduces the value of the indicator resulting in a better energy performance. Only the comparison with the baseline shows that the energy efficiency increases only slightly. Thus, the parallel aggregation of baselines and performance is essential to evaluate the performance of a complex under fluctuating production conditions.

\section{REFERENCES}

[1] Drumm, C.; Busch, J.; Dietrich, W.; Eickmans, J.; Jupke, A.: STRUCTese ${ }^{\circledR}$ - Energy efficiency management for the process industry. Chemical Engineering and Processing: Process Intensification 67, p. 99-110, 2013

[2] Windecker, G.; Krämer, S.: EnPls als wichtige Indikatoren für die Verbesserung der Energieeffizienz - Auswertung der NAMUR Umfrage des AK 4.17. Vortrag NAMUR-Hauptsitzung, 2014

[3] ISO 50001: Energy management. ISO 2011, http://www.iso.org

[4] Kalliski, M.; Krahé, D.; Beisheim, B.; Krämer, S.; Engell, S.: Resource efficiency indicators for real-time monitoring and optimization of integrated chemical production plants. Computer Aided Chemical Engineering 37, p. 1949-1954, 2015

[5] ISO/DIS 20140-5: Automation systems and integration - Evaluating energy efficiency and other factors of manufacturing systems that influence the environment - Part 5: Environmental influence evaluation data. ISO 2015, http://www.iso.org

[6] IEC TR 62837: Energy efficiency through automation systems. IEC 2013

\section{VISUALIZATION OF RESOURCE EFFICIENCY}

In the MORE project, we have developed efficient dashboard concepts for the visualization of multi-dimensional REI information. Such dashboards require elements that are suited to display complex information and can still be easily interpreted. To go beyond the possibilities of classic two- or three-dimensional representations, the use of additional attributes like colour, orientation, size and a smart combination of specialized visualization elements leads to dashboard solutions that display the contributions to the overall resource efficiency. Figure 7 shows a dashboard developed for an Acrylonitrile plant operated by Ineos Köln (numbers randomly generated) including a control panel for the navigation through the plant hierarchy with efficiency indicator bars for three plant sections indicating the overall efficiency (left). On user selection, historical trends for the selected sub-section and resource-specific REIs are displayed as aggregated tiles (right). The current value and its trend are shown by the colour and the orientation of the associated arrows next to the latest tile.

For the example shown in Figure 7, the user can identify a deficiency in the plant section P1 that is caused by the inefficient use of steam and an elevated by-product concentration in the product stream. Moreover, the plant operator can navigate down the plant hierarchy of P1 to investigate the situation further by means of a variety of different diagrams that are accessible via a drop down menu (bottom-left).

\section{STANDARDISATION}

The MORE project is committed to the standardisation of the methodology for the definition of real-time resource efficiency indicators to guide industrial users during the development of REIs for their applications. The ultimate goal is an internationally recognised "state of the art" document that disseminates the methodology within the process management community. The Namur ad-hoc working group (AK) Resource Efficiency Indicators for the operational use is currently preparing a Namur Recommendation including a list of general REIs that are applicable across a wide range of industrial sectors. The standardisation plan given in figure 8 includes the intention to use the Namur Recommendation as a basis for an IEC Technical Report (TR). These results can complement current Cenelec, ISO, IEC and also national standardisation activities such as ISO 20140 - Part 5 Automation systems and integration - Evaluating energy efficiency and other factors of manufacturing systems that influence the environment [5] and IEC TR 62837 Energy efficiency through automation systems [6]. 


\section{CONCLUSIONS}

Real-time resource efficiency indicators as developed by the MORE project measure the efficiency of production processes over short periods accurately and unambiguously and have a large potential to help operations to improve the resource and energy efficiency of the production and to increase the competitiveness on globalised markets. The methodology enables the industrial users to establish a bottom-up approach to monitor and subsequently improve the material and energy efficiency. The efficient visualisation of the deviations from the current baseline focuses the attention of the operating staff to critical sections of the plant where a root-cause analysis should be performed. Furthermore, the REIs can be used as objectives in optimization-based operator advisory sys- tems and within advanced process control schemes to realise an online optimisation of energy and resource efficiency. The instigated standardisation efforts will help to establish a unified approach throughout the chemical and process industries in Europe.

\section{ACKNOWLEDGEMENTS}

The project MORE receives funding under the seventh framework programme of the EU No. 604068.

\section{AUTHORS}

MARC KALLISKI (born 1987) studied Chemical Engineering at the TU Dortmund University from 2007-2013. Since september 2013 he has been a research associate with the Chair of Process Dynamics and Operations at TU Dortmund, working on his PhD in the field of resource efficiency optimisation in batch processing plants and the visualisation of multi-dimensional resource efficiency indicators.

Technische Universität Dortmund,

Fakultät Bio- und Chemieingenieurwesen, Lehrstuhl für Systemdynamik und Prozessführung,

D-44221 Dortmund, Tel. +49 (0) 23175573 78,

E-Mail: Marc.Kalliski@bci.tu-dortmund.de

BENEDIKT BEISHEIM (born 1987) studied Chemical Engineering at the RWTH Aachen University from 2007-2014. Since May 2014 he has joined INEOS in Köln, working in the energy management department. He is responsible for energy optimization projects and is involved in the EU-funded projects MORE and DYMASOS.

DANIEL KRAHÉ (born 1983) studied Chemical Engineering at the TU Dortmund University and finished his studies in August 2013 with a diploma thesis. Since November 2013 he is employed the DYN group as a research assistant for the EU project MORE and focusses on decision support using multi-criterial real-time optimisation strategies.

Dr.-Ing. UDO ENSTE (born 1968) studied Electrical Engineering at the RWTH Aachen and was a research associate with the Chair of Process Control
Engineering, RWTH Aachen from 1995-2000. Since 2000 he has been managing director of LeiKon $\mathrm{GmbH}$. Since 1997 he has been a member of the normative standardisation group of DIN DKE K931 'System aspects in automation and process control' and since 2011 chairman of the German working group in Namur 'MES' (Manufacturing Execution Systems).

Dr.-Ing. STEFAN KRÄMER (born 1972) studied Chemical Engineering at the Universtiy of Newcastle upon Tyne (GB) and at TU Dortmund University. He joined INEOS in 2004 as an Advanced Control Engineer and ran a team of APC and DCS engineers from 2009 to 2013. As Site Energy Manager his responsibilities now include operating the site wide energy management system and coordinating site energy optimization and energy efficiency projects. He teaches Batch Process Operation as a Guest Lecturer at TU Dortmund University. He is active in the Namur working group on Energy Efficiency. In the FP7 project MORE he has the role of industrial application coordinator.

Prof. Dr.-Ing. SEBASTIAN ENGELL (born 1954) received the Dipl.-Ing. degree in Electrical Engineering from the Ruhr-Universität Bochum, Germany, in 1978, and the Dr.-Ing. degree from the Universität Duisburg, Germany, in 1981. In 1990, he was appointed to his present position as a Full Professor of process dynamics and operations with the Department of Biochemical and Chemical Engineering at TU Dortmund University. His areas of research are control design, modeling and control of chemical and biochemical processes, hybrid systems, logic control and scheduling in the process industries. 